\title{
TEMPO DE PERMANÊNCIA DO DISPOSITIVO VENOSO PERIFÉRICO, IN SITU, RELACIONADO AO CUIDADODE ENFERMAGEM, EM PACIENTES HOSPITALIZADOS
}

\author{
TIME OF PERMANENCE OF THE PERIPHERICAL VENOUS DEVICE IN SITU \\ RELATED TO THE NURSING CARE TO HOSPITALIZED PATIENTS
}

Renata Cristina de C. Pereira ${ }^{1}$; Maria Lúcia Zanetti² \& Kátia P. Ribeiro ${ }^{3}$

\begin{abstract}
${ }^{1}$ Aluna do sétimo semestre de graduação, bolsista do Programa Especial de Treinamento - Capes; ${ }^{2}$ Orientadora do projeto, Docente do Departamento de Enfermagem Geral e Especializada. Escola de Enfermagem de Ribeirão Preto da Universidade de São Paulo. ${ }^{3}$ Enfermeira, Bolsista de Apoio Técnico CNPq.

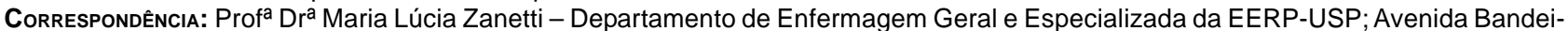
rantes, 3900 - Campus Universitário - CEP 14040-902 - Ribeirão Preto-SP - FAX: 633-3271/630-2561- zanetti@eerp.usp.br.
\end{abstract}

PEREIRA RCC; ZANETTI ML \& RIBEIRO KP. Tempo de permanência do dispositivo venoso periférico, in situ, relacionado ao cuidado de enfermagem, em pacientes hospitalizados. Medicina, Ribeirão Preto, 34: 79-84, jan./mar.2001.

RESUMO: O desenvolvimento tecnológico da terapia intravenosa impõe ao enfermeiro competência técnica e científica. Este estudo descritivo teve como objetivo relacionar o tempo de permanência in situ dos dispositivos venosos, periféricos com o cuidado de enfermagem. Os dados obtidos apontam que, em $50 \%$ das punções venosas, com tempo de permanência inferior a $24 \mathrm{~h}$, o grau de cuidado de enfermagem foi insatisfatório, tendo 9,4\% delas permanecido in situ por um período superior a $72 \mathrm{~h}$. O tempo médio de permanência do dispositivo venoso periférico, in situ foi de $29,2 \mathrm{~h}$, o que demonstra necessidade de intensificar estudos visando à qualificação do cuidado de enfermagem.

UNITERMOS: Infusões Endovenosas; complicações. Cuidados de Enfermagem.

\section{INTRODUÇÃO}

Concomitante ao avanço tecnológico da terapia intravenosa, o enfermeiro deve mostrar sua competência técnica e científica para o desenvolvimento da prática da punção de veias periféricas e administração de medicamentos, considerando-se que é responsabilidade da enfermagem a decisão sobre a escolha de locais, tipos de dispositivos, calibres, documentação da instalação, manutenção de curativos e prevenção de complicações.

Atualmente, o procedimento da terapia intravenosa tem sido alvo de pesquisas nos Estados Unidos e Europa, por ser um recurso vastamente utiliza- do na terapêutica medicamentosa do paciente e que pode gerar inúmeras complicações ${ }^{(1)}$.

A observação da punção é extremamente importante, pois, se o local de inserção mostrar-se com sinais e sintomas de complicações, a terapia deve ser suspensa, mesmo que o cateter in situ esteja instalado por um período inferior a 24 horas, e a atitude deve ser tomada pela equipe de enfermagem, que possui autonomia para tomar as decisões pertinentes a cada caso $^{(1)}$.

Segundo Maki \& Ringer $1991^{(2)}$, para a manutenção da terapia intravenosa, os princípios recomendados são: a observação diária do local da inserção, para redução dos riscos de flebite e infecções, e a 
rotatividade desses locais, com intervalos de 24 a $48 \mathrm{~h}$. Por outro lado, Bregenzer et al., $1998^{(3)}$, alertam para o desconforto que novas punções proporcionam ao paciente, prática que aumenta consideravelmente o custo da terapia intravenosa.

Ao avaliarem o tempo in situ dos dispositivos intravenosos, periféricos, Gonçalves et al., 1998 ${ }^{(4)}$, mostraram os benefícios da utilização de solução heparinizada ou água destilada esterilizada para a manutenção adequada do dispositivo periférico, principalmente para prevenir a formação de coágulos, evitando, assim, a sua obstrução. Relatam, ainda, que os dispositivos venosos periféricos, do tipo scalp e/ou jelco mantiveram-se pérvios, por um período de até $72 \mathrm{~h}$, com irrigação intermitente em intervalos de $6 / 6 \mathrm{~h}$ ou 12/12 h, com um volume de $2 \mathrm{ml}$ de água destilada, esterilizada ou solução heparinizada com 50UI/ml, na totalidade dos pacientes.

Entretanto, Lundgren et al., $1993^{(5)}$, apontam que o cuidado de enfermagem, relacionado à manutenção do dispositivo venoso periférico in situ, diminui com o passar dos dias e que, após o segundo dia de observação, tal cuidado foi considerado insatisfatório.

Pereira \& Zanetti, $2000^{(6)}$, ao investigarem pacientes cirúrgicos, internados e submetidos à terapia intravenosa, constataram que o grau do cuidado de enfermagem, em $60 \%$ das punções realizadas, apresentou-se como insatisfatório e muito insatisfatório, sendo o tempo de permanência do dispositivo in situ de 24 a 72 h, em 47\% dos pacientes. Assim, reforçaram a necessidade de investigações da temática e apontaram a importância do aprimoramento de programas de educação em serviço, relacionados à terapia intravenosa.

\section{OBJETIVO}

Relacionar o tempo de permanência in situ dos dispositivos venosos, periféricos, com o cuidado de enfermagem.

\section{MATERIAL E MÉTODOS}

\subsection{Delineamento do estudo}

Utilizou-se o método descritivo, segundo Polit \& Hungler, $1995^{(7)}$.

\subsection{Local de estudo}

Realizou-se na seção A, da Clínica Cirúrgica do Hospital das Clínicas da Faculdade de Medicina de Ribeirão Preto da Universidade de São Paulo (HCFMRP/USP), onde as especialidades são: Gastrocirurgia e Cirurgia Geral, com pacientes cirúrgicos, ali internados.

\subsection{Amostra}

Quarenta pacientes submetidos a procedimentos cirúrgicos, internados nas enfermarias da Clínica Cirúrgica, especialidades Gastrocirurgia e Cirurgia Geral, no período de 10 de janeiro a 10 de fevereiro de 1999.

\subsection{Critérios de inclusão}

O estudo contou com pacientes que iniciaram a inserção do dispositivo venoso, periférico, os quais eram capazes de responder questões referentes a sinais e sintomas de complicação venosa, pacientes que não vieram transferidos de outra seção do referido hospital ou de outras instituições de saúde, já tendo sido submetidos a terapia intravenosa prévia. Todos os pacientes eram adultos, ou seja, maiores de dezoito anos de idade.

\subsection{Variáveis do estudo}

Variáveis relacionadas ao cuidado de enfermagem (grau de cuidado) e ao tempo de permanência do dispositivo venoso in situ .

Os graus de cuidados de enfermagem foram definidos, segundo Lundgren et al., $1993^{(5)}$. São eles: Grau 0- Cuidado de enfermagem satisfatório: quando o dispositivo apresentou boa fixação e limpeza e o paciente não se referiu a dor; Grau 1- $C u i$ dado de enfermagem insatisfatório: quando o dispositivo estava fixado com perda de asas ou linha intravascular, ou seja, insatisfatoriamente fixado; curativo incompleto; o cateter deslocava-se visivelmente na veia, quando o paciente movia o braço; paciente referiu que o dispositivo estava perfurando a veia, sangue na parte externa do dispositivo ou no curativo e Grau 2- Cuidado de enfermagem muito insatisfatório: quando não houve fixação satisfatória do dispositivo, ou seja, somente o curativo mantinha o dispositivo no local; o dispositivo deslocava-se na veia e o paciente referiu que o dispositivo estava perfurando a veia e o local de inserção; o dispositivo estava in situ há mais de cinco dias.

Tempo de permanência do dispositivo venoso periférico in situ: considerou-se o tempo em horas de permanência do dispositivo in situ, sendo o período compreendido aquele entre o início e o término da utilização do dispositivo venoso. 


\subsection{Elaboração do instrumento de coleta de dados}

Construiu-se um roteiro sistematizado, considerando-se as variáveis do estudo, a experiência pessoal do pesquisador e dados dos estudos de Lundgren et al., $1993^{(5)}$, e Nascimento, $1994^{(1)}$. Esse instrumento, com quatro questões fechadas, contém as variáveis relacionadas ao cuidado de enfermagem e tempo de permanência do dispositivo venoso in situ.

\subsection{Validação do instrumento}

Solicitou-se a colaboração de três enfermeiras especialistas e da chefe da Divisão de Enfermagem do hospital citado, para apreciarem o instrumento quanto à clareza dos itens, facilidade de leitura, compreensão e forma de apresentação, tendo o estudo piloto sido realizado no período de 14 a 17 de dezembro de 1998, com quatro pacientes.

\subsection{Coleta de dados}

O próprio pesquisador coletou os dados através da observação direta, obtendo, primeiramente, a escala de cirurgia, informando-se, assim, com um dia de antecedência, sobre quais pacientes iriam para cirurgia. No dia da cirurgia, o pesquisador compareceu ao hospital, às cinco horas e quarenta e cinco minutos, para acompanhar a instalação da terapia intravenosa, anotando as observações no próprio instrumento, sem fazer perguntas ao funcionário que inseriu o dispositivo. Após o término do procedimento e a saída do funcionário da enfermaria, perguntava-se ao paciente se gostaria de fazer algum comentário a respeito da instalação do dispositivo venoso. O pesquisador permanecia junto ao paciente de cinco a trinta minutos, período que antecedia ao chamado para o centro cirúrgico. A seguir, realizava-se a observação de outros pacientes que haviam iniciado anteriormente a terapia intravenosa e ainda a mantinham. Todos os dias, verificava-se a prescrição médica, com o objetivo de informar-se sobre os pacientes que teriam a terapia interrompida, a fim de acompanhar o procedimento de interrupção.

Quando o pesquisador verificava que a punção encontrava-se em outro local que não o da punção original para cirurgia, indagava-se ao paciente o motivo da troca, horário e local da ocorrência. O primeiro período de observação durou das cinco horas e quarenta e cinco minutos às onze horas e trinta minutos. Porém, às quinze horas, o pesquisador retornava à seção, onde aguardava os pacientes que saíam do centro de recuperação anestésica, continuando a observação até às dezoito horas.

\subsection{Organização dos dados para análise}

Os dados contidos no formulário receberam códigos específicos, quando selecionamos cada variável que era transportada para uma planilha. Montamos a estrutura do banco de dados a partir das planilhas, sendo o mesmo formatado no programa Word (para Windows 95, versão 6.0). Para análise dos dados, utilizamos os valores mínimo e máximo, média, mediana e desvio-padrão de horas de permanência do dispositivo venoso in situ.

\subsection{Aspectos éticos}

Submeteu-se o estudo à análise do Comitê de Ética em Pesquisa, do hospital em estudo, que foi aprovado em 07 de janeiro de 1999. Esclarecemos aos sujeitos participantes os objetivos e a natureza da investigação e iniciamos a observação, após terem eles assinado o termo de consentimento.

\section{RESULTADOS}

Dos quarenta pacientes submetidos à punção venosa, internados no HCFMRP - USP, 24 (60\%) estavam internados em enfermarias de Cirurgia Geral e 16 (40\%) de Gastrocirurgia.

Em relação ao número de punções venosas realizadas, obtivemos que, em 40 pacientes internados, foram realizadas 75 punções venosas, sendo $57,5 \%$ deles submetidos a uma só punção; $22,5 \%$ a duas; $7,5 \%$ a três; $5 \%$ a quatro e $7,5 \%$ a cinco ou mais.

$\mathrm{O}$ cuidado de enfermagem, implementado durante o período em que o paciente se encontrava em terapia intravenosa, mostrou-nos que $56 \%$ e $4 \%$ referem-se aos graus 1 e 2, respectivamente, perfazendo um total de $60 \%$ (Figura 1).

Quanto ao tempo de permanência, verificamos que, em $43 \%$ dos pacientes, o dispositivo venoso permaneceu por um período menor que $24 \mathrm{~h} ; 36 \%$ de 24 a $48 \mathrm{~h}$ e $11 \%$ de 48 a $72 \mathrm{~h}$, sendo que apenas $10 \%$ ficaram mais de $72 \mathrm{~h}$ (Figura 2).

Quando relacionamos o tempo de permanência in situ em horas e os graus de cuidados de enfermagem (Tabela I), constatamos que, em 50\% das punções com dispositivos venosos, periféricos, em pacientes submetidos a procedimentos cirúrgicos, com tempo de permanência inferior a $24 \mathrm{~h}$, o grau de cuidado de enfermagem foi insatisfatório (Grau 1). 


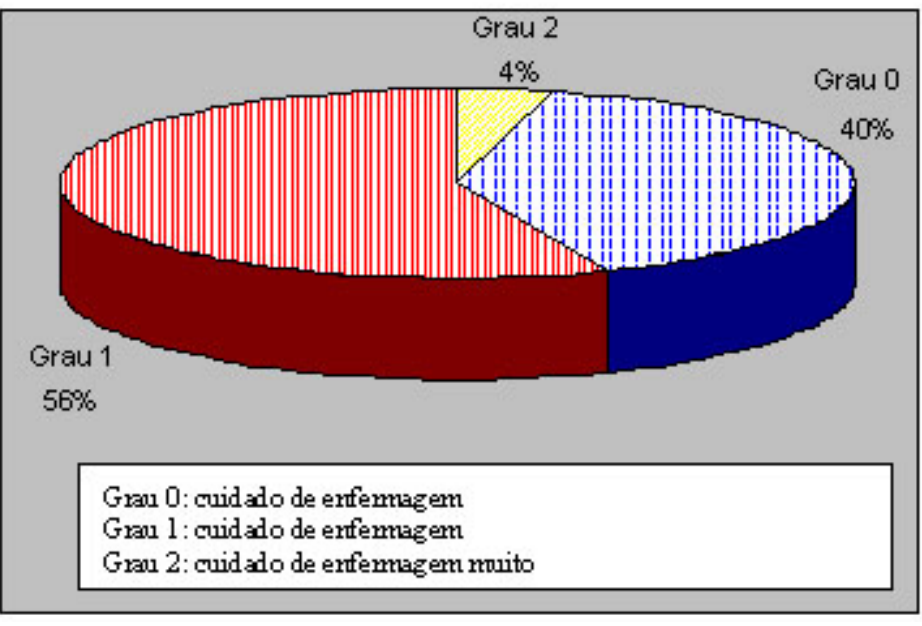

Figura 1 - Distribuição de freqüência das punções venosas, realizadas em pacientes internados em um hospital de grande porte, segundo o grau de cuidado de enfermagem (Ribeirão Preto - SP, 1999).

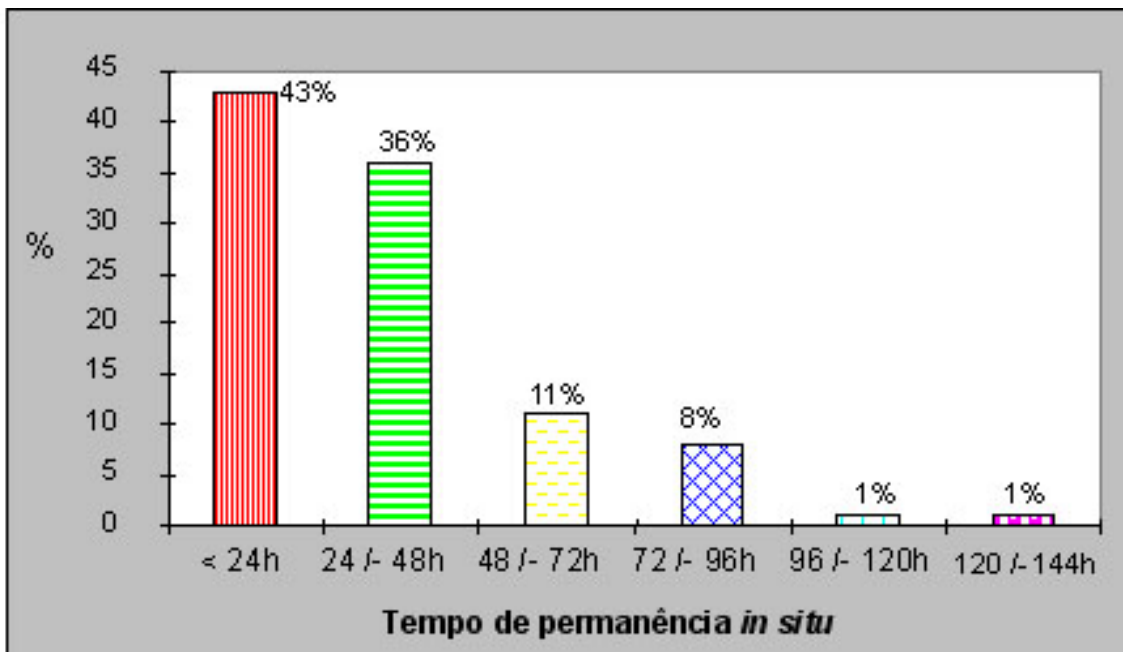

Figura 2 - Distribuição de freqüência de pacientes internados em um hospital de grande porte, segundo o tempo de permanência do dispositivo in situ (Ribeirão Preto - SP, 1999).

Verificamos que das 75 punções venosas, sete $(9,4 \%)$ permaneceram in situ por um período superior a $72 \mathrm{~h}$.

Analisando-se a Tabela II, que relaciona o tempo em horas de permanência do dispositivo venoso in situ com os graus de cuidados de enfermagem, obtivemos que o Grau 0 , variou de 0 a 78,8 h; a mediana $25,5 \mathrm{~h}$ e a média $25,3 \mathrm{~h}$ (desvio-padrão $=22 \mathrm{~h}$ ) de permanência do dispositivo venoso in situ. Quanto ao Grau 1, este variou de 0 a 105,9 h; a mediana $23,8 \mathrm{~h}$ e a média $27 \mathrm{~h}$ (desvio padrão $=25,5 \mathrm{~h}$ ) de permanência in situ. E, quando consideramos o Grau 2, cuidado de enfermagem muito insatisfatório, obtivemos variação de 80 a 136,2 h; a mediana 81,6 h e a média 99,3 h (desvio-padrão $=32 \mathrm{~h}$ ) de permanência do dispositivo venoso in situ.

\section{DISCUSSÃO}

Quanto ao número de punções, em $42,5 \%$ dos pacientes desta casuística foi realizada mais de uma punção. Fulton, $1997^{(8)}$, afirma que o número de inserções, o tipo de dispositivo e o tempo de duração constituemse fatores de risco para complicações em pacientes submetidos à terapia intravenosa. Assim, os resultados encontrados nesta investigação reforçam a necessidade de a equipe de enfermagem dispensar maior atenção aos critérios de escolha da rede venosa e aos cuidados subseqüentes para a sua manutenção.

Segundo Lundgren et al., 1993 ${ }^{(5)}$, o grau de cuidado de enfermagem pode ser alterado com o tempo de permanência do dispositivo in situ, sendo que, após o segundo dia de instalação, há comprometimento da qualidade do cuidado, como assinalado. O comprometimento pode estar associado à falta de observação de sinais e sintomas de possíveis complicações por parte da equipe de enfermagem. Os dados deste estudo apontam que $60 \%$ do cuidado de enfermagem, em relação à punção venosa, foram classificados como insatisfatórios e muito insatisfatórios. A situação poderia ser revertida através de padronização de condutas em relação à terapia intravenosa, diante de medidas como: observação sistemática, documentação no prontuário do paciente e comunicação entre a equipe, durante a passagem do plantão. Acreditamos que a qualidade do cuidado de enfermagem para tal procedimento podem ser alcançada através de estímulo, cabendo aos enfermeiros essa responsabilidade.

A literatura sobre o tempo adequado de permanência do dispositivo venoso, periférico in situ tem mostrado controvérsias, em relação ao tempo, variando de 48 a 72 horas ${ }^{(1,3,5,9)}$. Segundo Lundgren et al., $1993^{(5)}$, o enfermeiro deve observar atentamente os procedimentos, visando melhorar a qualidade do cuidado de enfermagem prestado, o tempo de permanência in situ e a boa fixação do dispositivo, para evitar complicações. Constatamos que os resultados ob- 


\begin{tabular}{|c|c|c|c|c|c|c|c|c|}
\hline \multirow{3}{*}{$\begin{array}{l}\text { Tempo de } \\
\text { permanência } \\
\text { (horas) }\end{array}$} & \multicolumn{6}{|c|}{ Cuidados de enfermagem } & & \\
\hline & \multicolumn{2}{|c|}{ Grau 0} & \multicolumn{2}{|c|}{ Grau 1} & \multicolumn{2}{|c|}{ Grau 2} & \multicolumn{2}{|c|}{ Total } \\
\hline & № & $\%$ & № & $\%$ & № & $\%$ & № & $\%$ \\
\hline$<24$ & 11 & 37 & 21 & 50 & - & - & 32 & 43 \\
\hline $24 /-48$ & 14 & 47 & 13 & 31 & - & - & 27 & 36 \\
\hline $48 /-72$ & 03 & 10 & 05 & 12 & - & - & 08 & 11 \\
\hline 72 /- 96 & 02 & 06 & 02 & 05 & 02 & 67 & 06 & 08 \\
\hline $96 /-120$ & - & - & 01 & 02 & - & - & 01 & 01 \\
\hline $120 /-144$ & - & - & - & - & 01 & 33 & 01 & 01 \\
\hline Total & 30 & 40 & 42 & 56 & 03 & 04 & 75 & 100 \\
\hline
\end{tabular}

\begin{tabular}{|c|c|c|c|c|c|}
\hline \multicolumn{6}{|c|}{$\begin{array}{l}\text { Tabela II - Valores mínimos e máximos, mediana, média e des- } \\
\text { vio-padrão em horas de permanência dos dispositivos venosos } \\
\text { periféricos in situ, segundo os graus de cuidados de enferma- } \\
\text { gem (Ribeirão Preto, 1999). }\end{array}$} \\
\hline $\begin{array}{l}\text { Cuidados de } \\
\text { enfermagem }\end{array}$ & $\begin{array}{c}\text { Valor } \\
\text { mínimo }\end{array}$ & $\begin{array}{l}\text { Valor } \\
\text { máximo }\end{array}$ & Mediana & Média & $\begin{array}{l}\text { Desvio } \\
\text { padrão }\end{array}$ \\
\hline Grau 0 & 0 & 78,83 & 25,55 & 25,30 & 22,44 \\
\hline Grau 1 & 0 & 105,90 & 23,88 & 27,03 & 25,50 \\
\hline Grau 2 & 80,02 & 136,25 & 81,65 & 99,30 & 32,00 \\
\hline Total & 0 & 136,25 & 25,15 & 29,23 & 28,16 \\
\hline
\end{tabular}

cuidado de enfermagem insatisfatório ocorreu somente após o segundo dia de permanência do dispositivo venoso in situ. Isso nos leva a ratificar que é necessário maior atenção da equipe de enfermagem, com relação à manutenção adequada das punções venosas, visto que o cuidado de enfermagem foi considerado, neste trabalho, insatisfatório na maioria das punções.

O tempo de manutenção da terapia intravenosa também depende das condições clínicas do paciente, tempo excedido a um período de 24 horas, na maioria dos casos. Durante a manutenção da terapia intravenosa, periférica, podem ocorrer falhas na infusão, impedindo que a terapia tenha continuidade numa mesma veia, sendo necessária nova inserção ${ }^{(10)}$. Nesse contexto, é imprescindível que a equipe de enfermagem dispense cuidado de enfermagem satisfatório a todos os pacientes submetidos à terapia intravenosa, a fim de poupá-los de sucessivas punções e das conseqüências associadas. $\mathrm{O}$ estudo realizado por Maki \& Ringer, $1991^{(2)}$, demonstrou que a colonização microbiana de cateteres venosos, periféricos aumenta, quando são deixados no mesmo local, por um período superior a 72 horas. Assim, Motta et al., 1999 (11), discutem a colonização microbiana em cateter venoso, periférico, apontando que sua contaminação ocorre através das mãos dos

tidos por nós, relacionados ao tempo de permanência, estão de acordo com os preconizados na literatura. No entanto, ao analisarmos os dados em conjunto com o grau de cuidado de enfermagem, verificamos que a situação é preocupante. Isto porque, considerando os resultados encontrados por Maki \& Ringer, $1991^{(2)}$, o tempo de permanência in situ por mais de 48 horas aumenta, aproximadamente, o risco de flebite em $30 \%$ e que, a partir de 72 horas, o risco eleva-se para 39 a 49\%. Daí a necessidade de a equipe de enfermagem estabelecer critérios rígidos a esse respeito e procurar desenvolver pesquisas, a fim de buscar subsídios que possam dar suporte às ações de enfermagem em relação à terapia intravenosa.

Estes achados divergem dos obtidos por Lundgren et al., $1993^{(5)}$, já que, naqueles, o grau de trabalhadores de saúde e pela flora cutânea normal do paciente. Ressaltam ainda que, em pacientes hospitalizados por longos períodos, a colonização da pele pode se alterar em decorrência da flora hospitalar, sendo a punção venosa um procedimento invasivo, que causa infecção no local da inserção do dispositivo venoso ou, então, infecção sistêmica (bacteremia). Os dados são preocupantes, visto que estudos prévios realizados por Maki \& Ringer, $1991^{(2)}$, e Brannen \& Surette, $1997^{(9)}$, recomendam que o tempo de permanência não exceda a 72 horas.

Os resultados desta pesquisa, em relação ao tempo de permanência in situ, segundo os graus de cuidados de enfermagem, mostram que a média de permanência do dispositivo venoso periférico foi de 29,2 horas (1753.8 minutos ). No entanto, os resulta- 
dos do estudo realizado por Hecker, $1989^{(12)}$, mostraram que a média da permanência do dispositivo venoso in situ foi de 74 horas (4440 minutos), enquanto Nascimento, 1994 ${ }^{(1)}$, obteve uma média de permanência de 40,0 horas (2401,4 minutos).

A média de permanência do dispositivo venoso in situ desta pesquisa mostrou-se inferior às de Hecker, $1989^{(12)}$, e Nascimento, $1994^{(1)}$, o que pode estar relacionado ao tipo de doença de base e ao material do dispositivo utilizado para a inserção, condições clínicas do paciente e qualificação profissional. Assim, há necessidade de intensificarmos os estudos sobre tal temática, estabelecendo novos critérios para possíveis comparações.

\section{CONCLUSÃO}

O conjunto de dados obtidos nesta investigação aponta que, em $50 \%$ das punções venosas com tempo de permanência inferior a 24 horas, o grau de cuidado de enfermagem foi insatisfatório. Em 9,4\%, as punções venosas permaneceram in situ por um período superior a 72 horas, sendo o tempo médio de permanência do dispositivo venoso periférico in situ de 29,2 horas. Assim, os dados encontrados merecem ser analisados pelos enfermeiros, visando ao estabelecimento de critérios e protocolos que possam dar suporte às ações de enfermagem em relação à terapia intravenosa.

PEREIRA RCC; ZANETTI ML \& RIBEIRO KP. Time of permanence of the peripherical venous device in situ related to the nursing care to hospitalized patients Medicina, Ribeirão Preto, 34: 79-84, jan./march 2001.

ABSTRACT: The technological development of the intravenous therapy imposes to the nurse technical-scientific ability for this practice. This study purposed to relate the permanence time in situ of the peripherical venous devices with the nursing care. The obtained data point that in $50 \%$ of the venous stipplers, with time of permanence less than $24 \mathrm{~h}$, the degree of nursing care was unsatisfactory, having $9.4 \%$ of them remained in situ for a period upper than $72 \mathrm{~h}$. The average of the time of permanence in situ of the peripherical venous device was $29,2 \mathrm{~h}$, what demonstrates necessity to intensify the studies on this thematic aiming at qualification of nursing care.

UNITERMS: Infusions, Intravenous; complications. Nursing Care.

\section{REFERÊNCIAS BIBLIOGRÁFICAS}

1 - NASCIMENTO E. Infusão intravenosa em veia periférica: associações entre motivos de interrupção e fatores envolvidos. Dissertação de Mestrado, Escola Paulista de Medicina da UNIFESP, São Paulo, p. 1- 91. 1994.

2 - MAKI DG \& RINGER M. Risk factors for infusion-related phlebitis with small peripheral venous catheters. Ann Intern Med 114: 845-854, 1991.

3 - BREGENZER T; CONEN D; SAKMANN P \& WIDMER AF. Is routine replacement of peripheral intravenous catheters necessary? Arch Intern Med 158: 151-156, 1998.

4 - GONÇALVES VCS; GUTIÉRREZ, MGR \& GLASHAN RQ. Manutenção de catéteres venosos periféricos de longa permanência com infusão intermitente. Acta Paul Enf 11: 79-91, 1998.

5 - LUNDGREN A; JORFELDT L \& EK AC. The care and handling of peripheral intravenous cannulae on 60 surgery and internal medicne patients: an observation study. J Adv Nurs 6: 963-971, 1993

6 - PEREIRA RCC \& ZANETTI ML. Complicações decorrentes da terapia intravenosa em pacientes cirúrgicos. Rev Latinoam Enf 8: 21-27, 2000
7 - POLIT DF \& HUNGLER BP. Fundamentos de pesquisa em enfermagem. Artes Médicas, Porto Alegre, 1995.

8 - FULTON J S. Long-term vascular access devices. Annu Rev Nurs Res 15: 237-262, 1997.

9 - BRANNEN S \& SURETTE D. Monitoring IV-site infections. Can Nurse 93: 49-50, 1997.

10 - NASCIMENTO E. Inserções intravenosas periféricas com catéter plástico. Análise do tempo de sobrevida em função do emprego de padrões e inovações tecnológicas. Tese de Doutorado, Escola Paulista de Medicina da UNIFESP. São Paulo, p. 1-137, 1999.

11 - MOTTA VA; JERÔNIMO MF; CUNHA ER, GRAZIANO KU; CAMARGO LF \& UIP DE. Estudo do crescimento microbiano das porções proximal e distal do cateter venoso periférico. Rev Soc Cardiol Est São Paulo 9: 1-7, 1999.

12 - HECKER JF. Failure of intravenous infusions from extravasation and phlebitis. Anaesth Intensive Care 17: 433439, 1989.

Recebido para publicação em 17/04/2000

Aprovado para publicação em 17/01/2001 